\title{
ERROR ANALYSIS OF A SPECTRAL PROJECTION OF THE REGULARIZED BENJAMIN-ONO EQUATION*
}

\author{
HENRIK KALISCH ${ }^{1, \star \star}$ \\ ${ }^{1}$ Department of Mathematics, NTNU, Trondheim 7491, Norway. \\ email:kalisch@math.ntnu.no
}

\begin{abstract}
.
The regularized Benjamin-Ono equation appears in the modeling of long-crested interfacial waves in two-fluid systems. For this equation, Fourier-Galerkin and collocation semi-discretizations are proved to be spectrally convergent. A new exact solution is found and used for the experimental validation of the numerical algorithm. The scheme is then used to study the interaction of two solitary waves.
\end{abstract}

AMS subject classification (2000): 35Q53, 65M12, 65M70.

Key words: spectral methods, collocation approximation, internal waves.

\section{Introduction.}

In this article, consideration is given to the error analysis of a spatial discretization of the regularized Benjamin-Ono equation

$$
u_{t}+u_{x}+\varepsilon u u_{x}+\varepsilon H u_{x t}=0,
$$

where $H$ denotes the Hilbert transform, and $\varepsilon$ is a small parameter related to some physical quantities appearing in the derivation of the equation. The equation is a model for the time evolution of long-crested waves at the interface between two immiscible fluids. It is approximately valid for long waves with small amplitude if one of the fluid layers has large depth and the other is very thin. Typical situations in which the equation is useful are the pycnocline in the deep ocean, and the two-layer system created be the inflow of fresh water from a river into the sea. The variable $u(x, t)$ denotes the deflection of the free interface from the rest position at the spatial point $x$ and at time $t$, and the $x$-axis points in the direction of propagation of the waves. The equation is formally equivalent to the Benjamin-Ono equation

$$
u_{t}+u_{x}+\varepsilon u u_{x}-\varepsilon H u_{x x}=0,
$$

* Received September 2004. Revised January 2005. Communicated by Uri Ascher.

$\star \star$ This work was supported in part by the BeMatA program of the Research Council of Norway. 
which was introduced by Benjamin [2], and later rederived by Ono [7] as a model equation in the same situation.

Equation (1.1) has appeared previously in [1] and [4], where it was proved that the equation is well posed globally in time in the Sobolev space $H^{s}$, when $s \geq 3 / 2$. It was also shown in [1] that solutions of the two equations with the same initial data stay close on a time scale long enough to observe nonlinear and dispersive effects.

Both equations admit so-called solitary-wave solution. These are profiles that decay to zero at infinity, and propagate without changing their shape over time. For the Benjamin-Ono equation, these solutions are given by an explicit formula which was found by Benjamin [2]. The solitary waves for (1.1) are given by a simple rescaling of the Benjamin-Ono solitary waves [4]. A related class of solutions which are known in closed form are the periodic traveling waves. For (1.2), these were also obtained by Benjamin [2]. Periodic traveling waves for the regularized Benjamin-Ono equation (1.1) are exhibited in the present article. To the best of knowledge of the author, explicit formulae for these solutions have not appeared previously.

In [4], a numerical approximation was used to indicate that Equation (1.1) does not constitute an infinite-dimensional completely integrable system. This was suggested by the observation that two solitary waves do not interact cleanly, but leave behind a small oscillatory tail. The discretization used in these computations was a Fourier-collocation approximation, coupled with a 4-stage RungeKutta time stepping method. The convergence of the numerical scheme was tested on some special solutions, but no proof of convergence was given. Therefore, it it our purpose now to demonstrate a proof of convergence of the space semi discretization put forward in [4]. The proof makes use of an argument appearing in the work of Maday and Quarteroni [6], where a spectral discretization of the Korteweg-deVries equation was proven to be convergent. The equation under study in the present paper appears simpler than the Korteweg-deVries equation because it can be written as an integral equation containing no net spatial derivatives (see (2.1)). Accordingly, a spatial discretization will result in a system of ordinary differential equations which is not stiff. This fact indicates that (1.1) might be more convenient in a modeling situation than (1.2), because it is better-suited for numerical integration.

In this paper, both the Fourier-Galerkin and the Fourier-collocation methods for the regularized Benjamin-Ono equation are proven to be spectrally convergent, although in practice only the collocation method is used. The Galerkin projection will be in focus in Section 2 while the collocation method will be treated in Section 3. In Section 4, an implementation of the collocation method is presented. The exact traveling-wave solutions are used to test the rate of convergence of the numerical scheme. Finally, the scheme is put to work to study the interaction of two solitary waves.

There has been some previous work on the error analysis of spectral discretizations of evolution equations for internal waves. The results of Pasciak [9] are for a family of equations similar, but not including the equation under study here. 
Moreover, the proof for the collocation projection is not given in detail. More recently, some work has been done on the Benjamin-Ono and related equations such as the intermediate-long wave equation by Pelloni and Dougalis [10, 11]. They analyze a fully discrete scheme using a Fourier-Galerkin projection for the spatial discretization. An interesting numerical scheme for the Benjamin-Ono equation has been advocated by Thomeé and Vasudeva Murthy [12]. Though not a spectral approximation, they nevertheless make use of the discrete Fourier transform in the evaluation of the convolution integral giving the Hilbert transform.

One of the reasons for deriving simplified models for wave motion in fluid surfaces or interfaces is the relative ease with which these model equations can be treated numerically. It is appealing that it can be proved that some of the numerical approximations in use are indeed convergent, as this in effect validates the rationale behind using these simple equations instead of the more complicated Euler equations from which they are extracted.

In the following, some notation will be established. We assume that the variables are nondimensional, and have been normalized to reach the tidy form

$$
u_{t}+u_{x}+u u_{x}+H u_{x t}=0 .
$$

On the real line $\mathbb{R}$, the Hilbert transform $H$ of a function $f$ is defined by the singular integral

$$
H f(x)=\text { p.v. } \frac{1}{\pi} \int_{-\infty}^{\infty} \frac{f(x-y)}{y} \mathrm{~d} y .
$$

Although the equation is originally posed on the real line, for the purpose of numerical approximation, the problem with periodic boundary conditions will be considered. The Hilbert transform of a periodic function with period $2 \pi$ is given by the principal value of another singular integral, namely

$$
H f(x)=\mathrm{p} \cdot \mathrm{v} \cdot \frac{1}{2 \pi} \int_{0}^{2 \pi} \cot \left(\frac{y}{2}\right) f(x-y) \mathrm{d} y .
$$

Alternatively, the Hilbert transform could be defined as a Fourier multiplier operator given by

$$
\widehat{H f}(k)=-i \operatorname{sgn}(k) \hat{f}(k) .
$$

The Fourier transform will be defined momentarily. Denote by $|\cdot|_{p}$ the $L^{p}$-norm on the interval $[0,2 \pi]$, given by

$$
|f|_{p}^{p}=\int_{0}^{2 \pi}|f(x)|^{p} \mathrm{~d} x
$$

for $1 \leq p<\infty$, and by

$$
|f|_{\infty}=\operatorname{ess} \sup _{x}|f(x)|
$$

if $p=\infty$. In particular, the $L^{2}$-norm can be defined by

$$
|f|_{2}^{2}=(f, f)
$$


where the inner product $(\cdot, \cdot)$ is given by

$$
(f, g)=\int_{0}^{2 \pi} f(x) \overline{g(x)} \mathrm{d} x,
$$

for functions $f$ and $g$ which have finite $L^{2}$-norm. Denote by $\|\cdot\|_{s}$ the Sobolev norm, given by

$$
\|f\|_{s}^{2}=\sum_{k \in \mathbb{Z}}\left(1+|k|^{2}\right)^{s}|\hat{f}(k)|^{2},
$$

where the Fourier coefficients $\hat{f}(k)$ of the function $f$ are defined by

$$
\hat{f}(k)=\frac{1}{2 \pi} \int_{0}^{2 \pi} \mathrm{e}^{-i k x} f(x) \mathrm{d} x .
$$

Recall the inversion formula

$$
f(x)=\sum_{k \in \mathbb{Z}} \mathrm{e}^{i k x} \hat{f}(k),
$$

and the convolution formula

$$
(\hat{f} * \hat{g})(k)=\widehat{f g}(k),
$$

where the convolution of two functions $\hat{f}$ and $\hat{g}$ on $\mathbb{Z}$ is formally defined by

$$
(\hat{f} * \hat{g})(k)=\sum_{m+n=k} \hat{f}(m) \hat{g}(n) .
$$

The subspace of $L^{2}$ spanned by the set

$$
\left\{\mathrm{e}^{i k x} \mid k \in \mathbb{Z},-\frac{N}{2} \leq k \leq \frac{N}{2}-1\right\}
$$

for $N$ even is denoted by $S_{N}$. In the following, it will always be assumed that $N$ is even. The operator $P_{N}$ denotes the orthogonal projection from $L^{2}$ onto $S_{N}$, defined by

$$
P_{N} f(x)=\sum_{-N / 2 \leq k \leq N / 2-1} e^{i k x} \hat{f}(k) .
$$

$P_{N}$ may also be characterized by the property that

$$
\int_{0}^{2 \pi}\left(P_{N} f-f\right) \phi \mathrm{d} x=0,
$$

for all $\phi \in S_{N}$ and for all $f \in L^{2}$.

The space of $n$ times continuously differentiable periodic functions on $[0,2 \pi]$ is denoted by $C_{\sharp}^{n}(0,2 \pi)$, while $C_{\sharp}^{0}(0,2 \pi)$ stands for the space of continuous periodic functions. The space of periodic Sobolev functions on the interval $[0,2 \pi]$ is defined as the closure of the space of smooth periodic functions $C_{\sharp}^{\infty}(0,2 \pi)$ with 
respect to the $H^{s}$-norm, and is denoted by $H_{\sharp}^{s}=H_{\sharp}^{s}(0,2 \pi)$. For $f \in H_{\sharp}^{m}$, the estimates

$$
\begin{aligned}
\left\|f-P_{N} f\right\|_{0} & \leq C_{P} N^{-m}\left|\partial_{x}^{m} f\right|_{2}, \\
\left\|f-P_{N} f\right\|_{n} & \leq C_{P} N^{n-m}\left|\partial_{x}^{m} f\right|_{2}
\end{aligned}
$$

hold for an appropriate constant $C_{P}$ (cf. [3]). Note that while the Sobolev spaces can be defined for any real number $s$, the error estimates are generally written for positive integer values which are then denoted by $m$. The space of continuous functions from the interval $[0, T]$ into the space $H_{\sharp}^{s}$ is denoted by $C\left([0, T], H_{\sharp}^{s}\right)$. Similarly, we also consider the space $C\left([0, T], S_{N}\right)$, where the topology on $S_{N}$ can be given by any norm. Finally note the inverse inequality

$$
\left|\partial_{x}^{n} \phi\right|_{0} \leq N^{n}\|\phi\|_{0}
$$

for integers $n>0$ and $\phi \in S_{N}$.

The periodic initial value problem associated to Equation (1.3) is

$$
\left\{\begin{array}{l}
u_{t}+u_{x}+u u_{x}+H u_{x t}=0, \quad x \in[0,2 \pi], t \geq 0 \\
u(x, 0)=u_{0}(x), \\
u(0, t)=u(2 \pi, t), \quad t \geq 0
\end{array}\right.
$$

Existence, uniqueness and continuous dependence on the initial data of solutions to (1.8) pose no special difficulty, and can be established in much the same way as in [4], where this was done for the initial-value problem on the real line. Shorttime existence can be proved using a contraction argument, and a global solution is obtained via a priori estimates. The results are summarized in the following theorem.

Theorem 1.1. Suppose $s \geq 3 / 2$, and $u_{0} \in H_{4}^{s}(0,2 \pi)$. For any $T>0$, there exists a solution $u \in C\left([0, T], H_{\sharp}^{s}\right)$ of (1.8). Moreover, there is a constant $\kappa$ depending on $T$ and $\left\|u_{0}\right\|_{s}$, such that $u$ satisfies the estimate

$$
\sup _{t \in[0, T]}\|u(\cdot, t)\|_{s} \leq \kappa .
$$

\section{The Fourier-Galerkin method.}

In this section, the Fourier-Galerkin method is presented and a proof of convergence given. Note that the equation in (1.8) is formally equivalent to the integral equation

$$
u_{t}=K\left(u+\frac{1}{2} u^{2}\right),
$$

where $K$ is a Fourier multiplier operator with the symbol

$$
\widehat{K f}(k)=\frac{-i k}{1+|k|} \hat{f}(k) .
$$


A space-discretization of (1.8) is defined as follows. Find a function $u_{N}$ from $[0, T]$ to $S_{N}$ which satisfies

$$
\left\{\begin{array}{l}
\left(\partial_{t} u_{N}+\partial_{x} u_{N}+\frac{1}{2} \partial_{x}\left(u_{N}^{2}\right)+\partial_{x} \partial_{t} H u_{N}, \phi\right)=0, \quad t \in[0, T] \\
u_{N}(0)=P_{N} u_{0}
\end{array}\right.
$$

for all $\phi \in S_{N}$. Since for each $t, u_{N}(\cdot, t) \in S_{N}, u_{N}$ has the form

$$
u_{N}(x, t)=\sum_{k=-\frac{N}{2}}^{\frac{N}{2}-1} \hat{u}_{N}(k, t) \mathrm{e}^{i k x}
$$

where $\hat{u}_{N}(k, t)$ are the Fourier coefficients of $u_{N}(\cdot, t)$. Since the discrete differentiation operator commutes with the projection operator $P_{N}$, we continue to denote it by $\partial_{x}$. An equivalent characterization of $u_{N}$ uses the formulation of (1.8) as an integral equation.

$$
\left\{\begin{array}{l}
\left(\partial_{t} u_{N}-K u_{N}-\frac{1}{2} K\left(u_{N}^{2}\right), \phi\right)=0, \quad t \in[0, T] \\
u_{N}(0)=P_{N} u_{0}
\end{array}\right.
$$

for all $\phi \in S_{N}$.

Since functions in $S_{N}$ are smooth, it can be seen immediately that the two formulations are equivalent. Equation (2.3) is useful for proving local existence of a solution as well as error estimates, while (2.2) can be exploited to derive $a$ priori estimates. Taking $\phi=\mathrm{e}^{i k x}$ for $-N / 2 \leq k \leq N / 2-1$ in (2.3) yields the following system of equations for the Fourier coefficients of $u_{N}$.

$$
\left\{\begin{array}{l}
\partial_{t} \hat{u}_{N}(k, t)=\frac{-i k}{1+|k|}\left[\hat{u}_{N}(k, t)+\frac{1}{2}\left(\hat{u}_{N} * \hat{u}_{N}\right)(k, t)\right] \\
\hat{u}_{N}(k, 0)=\hat{u}_{0}(k)
\end{array}\right.
$$

for $-N / 2 \leq k \leq N / 2-1$.

For the initial-value problem (2.4), it is straightforward to prove the existence of a local solution as (2.4) represents a system of coupled first-order ordinary differential equations. The following theorem is proved using the contraction mapping principle. Since the argument is standard, the proof is omitted here.

THEOREM 2.1. The initial-value problem (2.4) has a unique real solution in the space $C\left(\left[0, t_{0}\right], S_{N}\right)$ for some positive time $t_{0}$.

In order to continue the solution to a prescribed time interval $[0, T]$, a priori estimates on $\left\|u_{N}\right\|_{s}$ are used. For the problem (1.8), an a priori bound is available for the $H^{\frac{3}{2}}$-norm. As it turns out, a similar estimate is also available for the discrete solution. The first step is to prove the conservation of a quantity related to the $H^{\frac{1}{2}}$-norm of $u_{N}$. 
TheOREM 2.2. Suppose $u_{N} \in C\left([0, T], S_{N}\right)$ is a solution of $(2.2)$. Then the following two equations hold.

$$
\begin{aligned}
& \frac{\mathrm{d}}{\mathrm{d} t}\left\{\int_{0}^{2 \pi} u_{N}(x, t) \mathrm{d} x\right\}=0, \\
& \frac{\mathrm{d}}{\mathrm{d} t}\left\{\int_{0}^{2 \pi} u_{N}^{2}(x, t) \mathrm{d} x+\int_{0}^{2 \pi} u_{N}(x, t) \partial_{x} H u_{N}(x, t) \mathrm{d} x\right\}=0 .
\end{aligned}
$$

Proof. To prove (2.5), use $1 \in S_{N}$ as a test function in (2.2) to obtain

$$
\int_{0}^{2 \pi} \partial_{t} u_{N}(x, t) \mathrm{d} x=\int_{0}^{2 \pi}\left(\partial_{x} u_{N}(x, t)+\frac{1}{2} \partial_{x}\left(u_{N}^{2}\right)(x, t)+\partial_{x} \partial_{t} H u_{N}(x, t)\right) \mathrm{d} x .
$$

From the periodicity and the fact that $u_{N}$ is smooth, it follows that the integral on the right-hand side is zero. Again using that $u_{N}$ is smooth, we can interchange the integral and the time derivative in the term on the left, and it is plain that (2.5) holds true. To prove (2.6), use $u_{N} \in S_{N}$ as a test function in (2.2) to obtain

$$
\left(\partial_{t} u_{N}+\partial_{x} \partial_{t} H u_{N}, u_{N}\right)=-\left(\partial_{x} u_{N}, u_{N}\right)-\frac{1}{2}\left(\partial_{x}\left(u_{N}^{2}\right), u_{N}\right) .
$$

It is readily seen that the right-hand side is zero. Rewriting the left-hand side gives (2.6).

Corollary 2.3. Suppose $s \geq 3 / 2$, and $u_{0} \in H_{\sharp}^{s}(0,2 \pi)$. For any $T>0$, there exists a solution $u_{N} \in C\left([0, T], H_{\sharp}^{s}\right)$ of (2.4). Moreover, there is a constant $\kappa$ depending on $T$ and $\left\|u_{0}\right\|_{s}$, such that

$$
\sup _{t \in[0, T]}\left\|u_{N}(\cdot, t)\right\|_{s} \leq \kappa
$$

The proof of this corollary follows the same lines as the proof for the initialvalue problem on the real line $\mathbb{R}$ in [4]. The conservation of the $H^{\frac{1}{2}}$-norm is used to bootstrap up to a bound on the $H^{s}$-norm for $s \geq 3 / 2$. For the sake of simplicity, the same constant $\kappa$ as in (1.9) is used. This bound allows us to continue the local solution by reapplying the contraction argument a finite number of times to obtain a solution $u_{N}$ on the fixed time interval $[0, T]$. Having shown the existence of such a solution for a fixed $N$, we turn to the proof of convergence of the Galerkin scheme.

TheOREm 2.4. Suppose $u_{0} \in H_{\sharp}^{m}(0,2 \pi)$ for $m \geq 2$. Given $T>0$ and $N \in \mathbb{Z}_{+}$, there is a unique solution $u_{N}$ to the finite-dimensional problem (2.2). Moreover, there exists a constant $\Lambda$, such that

$$
\sup _{t \in[0, T]}\left\|u(\cdot, t)-u_{N}(\cdot, t)\right\|_{0} \leq \Lambda N^{-m} .
$$


Proof. The existence and uniqueness of a solution for a fixed $N$ on the time interval $[0, T]$ has already been shown. To prove the convergence of the solution of the semi-discrete problem to a solution of the continuous problem, suppose $u_{N}$ is a solution of (2.2) and $u$ is a solution of (1.8). Let $h=P_{N} u-u_{N}$. Since $K$ commutes with the projection operator $P_{N}$, it follows that $h$ satisfies the relation

$$
\left(h_{t}, \psi\right)=(K h, \psi)-\frac{1}{2}\left(K\left(u_{N}^{2}\right), \psi\right)+\frac{1}{2}\left(P_{N} K\left(u^{2}\right), \psi\right), \quad \text { for all } \psi \in S_{N} .
$$

Choosing $h \in S_{N}$ as a test function, and then using the fact that $K$ is bounded and skew-symmetric on $L^{2}$ gives

$$
\begin{aligned}
\frac{1}{2} \frac{\mathrm{d}}{\mathrm{d} t}\|h\|_{0}^{2} & =(K h, h)+\frac{1}{2}\left(K\left(u^{2}\right), h\right)-\frac{1}{2}\left(K\left(u_{N}^{2}\right), h\right) \\
& \leq \frac{1}{2}\left\|K\left(u^{2}\right)-K\left(u_{N}^{2}\right)\right\|_{0}\|h\|_{0} \\
& \leq \frac{1}{2}\left\|u^{2}-u_{N}^{2}\right\|_{0}\|h\|_{0} .
\end{aligned}
$$

Finally, using the a priori estimates (1.9) and (2.7), we obtain

$$
\begin{aligned}
\frac{\mathrm{d}}{\mathrm{d} t}\|h\|_{0} & \leq \frac{1}{2}\left|u+u_{N}\right|_{\infty}\left\|u-u_{N}\right\|_{0} \\
& \leq \frac{1}{2}\left\|u+u_{N}\right\|_{m}\left\|u-P_{N} u+P_{N} u-u_{N}\right\|_{0} \\
& \leq \kappa\left\|u-P_{N} u\right\|_{0}+\kappa\left\|P_{N} u-u_{N}\right\|_{0} \\
& \leq \kappa\left\|u-P_{N} u\right\|_{0}+\kappa\|h\|_{0} .
\end{aligned}
$$

Using (1.5) and (1.9), it can be seen that

$$
\frac{\mathrm{d}}{\mathrm{d} t}\|h\|_{0} \leq \kappa C_{P} N^{-m} \kappa+\kappa\|h\|_{0} .
$$

Since $h(\cdot, 0)=0$, Gronwall's inequality may be used to show that there is a constant $\lambda$, depending on $T$ and $\left\|u_{0}\right\|_{m}$, such that

$$
\sup _{t \in[0, T]}\|h(\cdot, t)\|_{0} \leq \lambda N^{-m} .
$$

For a fixed $t \in[0, T]$, the error between $u$ and $u_{N}$ can now be estimated using the triangle inequality, (1.5) and (2.8) as follows.

$$
\begin{aligned}
\left\|u(\cdot, t)-u_{N}(\cdot, t)\right\|_{0} & \leq\left\|u(\cdot, t)-P_{N} u(\cdot, t)\right\|_{0}+\left\|P_{N} u(\cdot, t)-u_{N}(\cdot, t)\right\|_{0} \\
& \leq C_{P} N^{-m}\|u(\cdot, t)\|_{m}+\|h(\cdot, t)\|_{0} \\
& \leq C_{P} \kappa N^{-m}+\lambda N^{-m} .
\end{aligned}
$$

On taking the supremum in $t$ and setting $\Lambda=C_{P} \kappa+\lambda$, it follows that

$$
\sup _{t \in[0, T]}\left\|u(\cdot, t)-u_{N}(\cdot, t)\right\|_{0} \leq \Lambda N^{-m}
$$

It appears to be more convenient, to have a convergence result in the $H^{1}$ norm, as this will also yield convergence in $L^{\infty}$. Using the inverse inequality (1.7) and (2.8), the following theorem can be proved. 
TheOREM 2.5. Suppose $u_{0} \in H_{\sharp}^{m}(0,2 \pi)$ for $m \geq 2$. Given $T>0$ and $N \in \mathbb{Z}_{+}$, there is a unique solution $u_{N}$ to the finite-dimensional problem (2.2). Moreover, there exists a constant $\tilde{\Lambda}$, such that

$$
\sup _{t \in[0, T]}\left\|u(\cdot, t)-u_{N}(\cdot, t)\right\|_{1} \leq \tilde{\Lambda} N^{1-m}
$$

This theorem concludes the section on the Fourier-Galerkin approximation. Note that the order of convergence of the scheme depends on the regularity of the initial data $u_{0}$. In particular, for smooth data the scheme converges spectrally, i.e. faster than any rational function of $N$. Although the preceding theorems seem satisfactory, it turns out that the Fourier-Galerkin method is rarely used in practice. Instead, the most widely used spectral projection is the pseudo-spectral method, where nonlinear terms are evaluated in physical space. The advantage of this approach is the utilization of the Fast Fourier Transform to evaluate the nonlinear terms. Since this method is equivalent to the Fourier-collocation method, it is imperative to have a convergence result for the Fourier-collocation method. Such a theorem will be provided in the next section.

\section{The Fourier-collocation method.}

In order to obtain a collocation projection of Equation (1.1), one may first define the interpolation operator $I_{N}$. Let the collocation points be $x_{j}=2 \pi j / N$ for $j=0,1, \ldots, N-1$. Then, given $u \in C_{\sharp}^{0}(0,2 \pi), I_{N} u$ is the unique element in $S_{N}$, such that $I_{N} u\left(x_{j}\right)=u\left(x_{j}\right)$ for $j=0,1, \ldots, N-1 . I_{N} u$ is also called the $N$-th trigonometric interpolant of $u$. It has been proved in $[5,8]$ that when $u \in H_{\sharp}^{m}(0,2 \pi)$ with $m \geq 1$, there exists a constant $C_{I}$, such that

$$
\left\|u-I_{N} u\right\|_{0} \leq C_{I} N^{-m}\left|\partial_{x}^{m} u\right|_{2},
$$

and more generally

$$
\left\|u-I_{N} u\right\|_{n} \leq C_{I} N^{n-m}\left|\partial_{x}^{m} u\right|_{2} .
$$

The discrete derivative in the collocation approximation is defined by

$$
\partial_{N}=\partial_{x} I_{N}
$$

Note that the derivative $\partial_{x}$ does not commute with the interpolation operator. The discrete form of the operator $K$ is defined analogously by

$$
K_{N}=K I_{N}
$$

Finally, the collocation approximation to (1.8) is given by a function $v_{N}$ from $[0, T]$ to $S_{N}$, such that

$$
\left\{\begin{array}{l}
\left(\partial_{t} v_{N}+\partial_{N} v_{N}+\frac{1}{2} \partial_{N}\left(v_{N}^{2}\right)+\partial_{N} \partial_{t} H v_{N}\right)\left(x_{j}\right)=0, \quad t \in[0, T] \\
v_{N}(0)=I_{N} u_{0}
\end{array}\right.
$$


for $j=0,1,2, \ldots, N-1$. Thus we assume that the solution is written as the sum

$$
v_{N}(x, t)=\sum_{k=-\frac{N}{2}}^{\frac{N}{2}-1} \tilde{v}_{N}(k, t) \mathrm{e}^{i k x}
$$

where the $\tilde{v}_{N}(k, t)$ can be thought of as the discrete Fourier coefficients of $v_{N}(x, t)$. Taking the discrete semi-inner product on the space $C^{0}(0,2 \pi)$ to be

$$
(\phi, \psi)_{N}=\frac{2 \pi}{N} \sum_{j=0}^{N-1} \phi\left(x_{j}\right) \overline{\psi\left(x_{j}\right)}
$$

we recall that for functions $\phi, \psi \in S_{N}$, this inner product is equal to the $L^{2}$-inner product

$$
(\phi, \psi)_{N}=(\phi, \psi)
$$

Using the semi-inner product $(\cdot, \cdot)_{N}$, one may define an equivalent approximation by requiring that

$$
\left\{\begin{array}{l}
\left(\partial_{t} v_{N}, \phi\right)_{N}=\left(K_{N}\left(v_{N}+\frac{1}{2} v_{N}^{2}\right), \phi\right)_{N}, \quad t \in[0, T] \\
v_{N}(0)=I_{N} u_{0} \in S_{N}
\end{array}\right.
$$

for all $\phi \in S_{N}$. Finally note that for $\phi \in S_{N}$, we have $K \phi=K_{N} \phi$. It follows that $K_{N}$ is skew-symmetric with respect to the inner product $(\cdot, \cdot)_{N}$.

The existence of a solution $v_{N}$ on a short time interval can be proved just as for the Galerkin approximation. In fact, the resulting equations for the Fourier coefficients of $v_{N}$ differ only in the nonlinear term. However, the time-span of existence of the solution might depend on $N$ as well as on the size of the initial data. In the Galerkin approximation, the conservation of the $H^{\frac{1}{2}}$-norm saved the day. For the collocation approximation, only the average $\int u_{N}(x, t) \mathrm{d} x$ is conserved, but this fact is not useful to gain an a priori estimate. Nevertheless, one can prove the following lemma which guarantees the existence of the approximate solution $v_{N}$ on a short time interval which is independent of $N$.

LEMma 3.1. For $\left\|v_{0, N}\right\|_{1}<R$, there exists $t^{*}$ only depending on $R$, such that there is a solution to (3.4) on the time interval $\left[0, t^{*}\right)$.

PROOF. As was mentioned, a local solution can be obtained using the standard theory of first-order systems of ordinary differential equations. We will prove an estimate of the form

$$
\frac{\mathrm{d}}{\mathrm{d} t}\left\|v_{N}\right\|_{1}^{2} \leq c\left\|v_{N}\right\|_{1}^{3}
$$

This suffices to continue the solution on to the interval $\left[0, t^{*}\right]$ for some $t^{*}$. By choosing $\phi=v_{N}$ in (3.3), the following equation is obtained.

$$
\begin{aligned}
\left(\frac{\mathrm{d}}{\mathrm{d} t} v_{N}, v_{N}\right)_{N} & =\left(K_{N} v_{N}, v_{N}\right)_{N}+\frac{1}{2}\left(K_{N}\left(v_{N}^{2}\right), v_{N}\right)_{N} \\
& =-\frac{1}{2}\left(I_{N}\left(v_{N}^{2}\right), K v_{N}\right) .
\end{aligned}
$$


Using the basic inequality

$$
|f|_{\infty} \leq c_{0}|f|_{2}^{1 / 2}\left|\partial_{x} f\right|_{2}^{1 / 2}
$$

it follows that we get the inequality

$$
\begin{aligned}
\frac{\mathrm{d}}{\mathrm{d} t}\left\|v_{N}\right\|_{0}^{2} & \leq\left\|I_{N}\left(v_{N}^{2}\right)\right\|_{0}\left\|K v_{N}\right\|_{0} \\
& \leq\left\|I_{N}\left(v_{N}^{2}\right)-v_{N}^{2}\right\|_{0}\left\|K v_{N}\right\|_{0}+\left\|v_{N}^{2}\right\|_{0}\left\|K v_{N}\right\|_{0} \\
& \leq C_{I}\left\|v_{N}^{2}\right\|_{0}\left\|v_{N}\right\|_{0}+\left\|v_{N}^{2}\right\|_{0}\left\|v_{N}\right\|_{0} \\
& \leq\left|v_{N}\right|_{\infty}\left(C_{I}+1\right)\left\|v_{N}\right\|_{0}^{2} \\
& \leq c_{0}\left(C_{I}+1\right)\left\|v_{N}\right\|_{0}^{1 / 2}\left\|\partial_{x} v_{N}\right\|_{0}^{1 / 2}\left\|v_{N}\right\|_{0}^{2} .
\end{aligned}
$$

Next choose $\phi=\partial_{x}^{2} v_{N}$ to obtain the equation

$$
\begin{aligned}
\left(\frac{\mathrm{d}}{\mathrm{d} t} v_{N}, \partial_{x}^{2} v_{N}\right)_{N} & =-\frac{1}{2}\left(I_{N}\left(v_{N}^{2}\right), K \partial_{x}^{2} v_{N}\right) \\
& =\frac{1}{2}\left(\partial_{x} I_{N}\left(v_{N}^{2}\right), K \partial_{x} v_{N}\right) .
\end{aligned}
$$

It follows analogously that

$$
\begin{aligned}
\frac{\mathrm{d}}{\mathrm{d} t}\left\|\partial_{x} v_{N}\right\|_{0}^{2} & \leq\left\|\partial_{x}\left(I_{N}\left(v_{N}^{2}\right)-v_{N}^{2}\right)\right\|_{0}\left\|K \partial_{x} v_{N}\right\|_{0}+\left\|\partial_{x}\left(v_{N}^{2}\right)\right\|_{0}\left\|K \partial_{x} v_{N}\right\|_{0} \\
& \leq\left\|I_{N}\left(v_{N}^{2}\right)-v_{N}^{2}\right\|_{1}\left\|v_{N}\right\|_{1}+2\left\|v_{N} \partial_{x} v_{N}\right\|_{0}\left\|v_{N}\right\|_{1} \\
& \leq C_{I}\left\|\partial_{x}\left(v_{N}^{2}\right)\right\|_{0}\left\|v_{N}\right\|_{1}+2\left|v_{N}\right|_{\infty}\left\|v_{N}\right\|_{1}\left\|v_{N}\right\|_{1} \\
& \leq 2 c_{0}\left(C_{I}+1\right)\left\|v_{N}\right\|_{0}^{1 / 2}\left\|v_{N}\right\|_{1}^{1 / 2}\left\|v_{N}\right\|_{1}^{2} .
\end{aligned}
$$

These two estimates combine to give an inequality of the form

$$
\frac{\mathrm{d}}{\mathrm{d} t}\left\|v_{N}\right\|_{1}^{2} \leq c\left\|v_{N}\right\|_{1}^{3} \text {. }
$$

Then a version of Gronwall's inequality yields the existence of a time $t^{*}>0$ and a constant $\beta^{*}>0$ depending on $R, c_{0}$ and $C_{I}$, such that

$$
\left\|v_{N}(\cdot, t)\right\|_{1} \leq \frac{\beta^{*}}{t^{*}-t}
$$

Using this estimate, it appears that the solution $u_{N}$ can be continued to the interval $\left[0, t^{*}\right)$ for each $N \in \mathbb{Z}_{+}$.

Since the global solution will be pieced together from a number of local solutions, it is convenient to define the following auxiliary problem, where the time $\tilde{t}$ will be defined in the next corollary.

$$
\left\{\begin{array}{l}
\left(\partial_{t} v_{N}+\partial_{N} v_{N}+\frac{1}{2} \partial_{N}\left(v_{N}^{2}\right)+\partial_{N} \partial_{t} H v_{N}\right)\left(x_{j}\right)=0, \quad t \in[n \tilde{t},(n+1) \tilde{t}] \\
v_{N}(n \tilde{t})=v_{N, 0}
\end{array}\right.
$$


for $j=0,1,2, \ldots, N-1$ and any integer $n \geq 0$. Since $c_{0}$ and $C_{I}$ are universal constants, the following corollary appears.

COROLlary 3.2. There exist $\tilde{t}<t^{*}$ and $\tilde{\beta}>0$ only depending on $R$, such that if $\left\|v_{N, 0}\right\|_{1} \leq R$, then a solution $v_{N}$ of $(3.6)$ exists on the interval $[n \tilde{t},(n+1) \tilde{t}]$, and

$$
\sup _{t \in[n \tilde{t},(n+1) \tilde{t}]}\left\|v_{N}(\cdot, t)\right\|_{1} \leq \tilde{\beta}
$$

The proof of this corollary is obvious. For instance, take $\tilde{t}=t^{*} / 2$ and $\tilde{\beta}=2 \beta^{*} / t^{*}$. Having in hand a local solution of the discrete problem, the next order of business is to establish an error estimate.

LEMMA 3.3. Suppose $v_{N}$ is a solution of (3.6) on the time interval $[n \tilde{t},(n+1) \tilde{t}]$, and

$$
\sup _{t \in[n \tilde{t},(n+1) \tilde{t}]}\left\|v_{N}(\cdot, t)\right\|_{1} \leq \tilde{\beta} .
$$

Then there are constants $\Lambda$ and $\lambda$, depending on $\|u(\cdot, n \tilde{t})\|_{m}, \tilde{t}, \tilde{\beta}$ and the final time $T$, such that

$$
\sup _{t \in[n \tilde{t},(n+1) \tilde{t}]}\left\|u(\cdot, t)-v_{N}(\cdot, t)\right\|_{0} \leq \Lambda N^{-m}+\lambda\left\|u(\cdot, n \tilde{t})-v_{N, 0}\right\|_{0} .
$$

Proof. Set $h=P_{N / 2} u-v_{N}$, where $P_{N / 2}$ is the Galerkin projection as defined in the previous section. Applying $P_{N / 2}$ to Equation (1.8) and subtracting from (3.4), we obtain the inequality

$$
\begin{aligned}
\frac{\mathrm{d}}{\mathrm{d} t}\|h\|_{0}^{2} & =\left(K P_{N / 2} u-K v_{N}, h\right)_{N}+\left(K_{N} P_{N / 2}\left(u^{2}\right)-K_{N}\left(v_{N}^{2}\right), h\right)_{N} \\
& =(K h, h)_{L^{2}}-\left(P_{N / 2}\left(u^{2}\right)-I_{N}\left(v_{N}^{2}\right), K h\right)_{N} \\
& =\left(I_{N}\left(v_{N}^{2}\right)-P_{N / 2}\left(u^{2}\right), K h\right) \\
& \leq\left\|I_{N}\left(v_{N}^{2}\right)-P_{N / 2}\left(u^{2}\right)\right\|_{0}\|h\|_{0} .
\end{aligned}
$$

Now observe that $\left(P_{N / 2} u\right)^{2} \in S_{N}$. It follows that

$$
\begin{aligned}
\left\|\left(P_{\frac{N}{2}} u\right)^{2}-I_{N}\left(v_{N}^{2}\right)\right\|_{0} & =\left\|I_{N}\left(\left(P_{\frac{N}{2}} u\right)^{2}-v_{N}^{2}\right)\right\|_{0} \\
& =\left(\left(P_{\frac{N}{2}} v\right)^{2}-v_{N}^{2},\left(P_{\frac{N}{2}} u\right)^{2}-v_{N}^{2}\right)_{N}^{1 / 2} \\
& \leq\left|P_{\frac{N}{2}} u+v_{N}\right|_{\infty}\left\|P_{\frac{N}{2}} u-v_{N}\right\|_{0} .
\end{aligned}
$$

Using the estimate $(3.5)$ and the fact that $\|u(\cdot, t)\|_{m} \leq \kappa$, it appears that

$$
\begin{aligned}
2 \frac{\mathrm{d}}{\mathrm{d} t}\|h\|_{0} & \leq\left\|P_{N / 2}\left(u^{2}\right)-u^{2}\right\|_{0}+\left\|u^{2}-\left(P_{N / 2} u\right)^{2}\right\|_{0}+\left\|\left(P_{N / 2} u\right)^{2}-I_{N}\left(v_{N}^{2}\right)\right\|_{0} \\
& \leq C_{P} 2^{m} N^{-m}\left\|u^{2}\right\|_{m}+\left|u+P_{\frac{N}{2}} u\right|_{\infty}\left\|u-P_{\frac{N}{2}} u\right\|_{0}+
\end{aligned}
$$




$$
\begin{aligned}
& +\left|P_{\frac{N}{2}} u+v_{N}\right|_{\infty}\left\|P_{\frac{N}{2}} u-v_{N}\right\|_{0} \\
\leq & C_{P} 2^{m} N^{-m}\|u\|_{m}^{2}+\left(c_{0}\|u\|_{1}+c_{0}\left\|P_{\frac{N}{2}} u\right\|_{1}\right) C_{P} 2^{m} N^{-m}\|u\|_{m}+ \\
& +\left(c_{0}\left\|P_{\frac{N}{2}} u\right\|_{1}+c_{0}\left\|v_{N}\right\|_{1}\right)\left\|P_{\frac{N}{2}} u-v_{N}\right\|_{0} .
\end{aligned}
$$

Hence,

$$
\begin{aligned}
\frac{\mathrm{d}}{\mathrm{d} t}\|h\|_{0} & \leq C_{P} 2^{m-1} N^{-m} \kappa^{2}+c_{0} \kappa C_{P} 2^{m} N^{-m} \kappa+\frac{1}{2} c_{0}\left(\kappa+\left\|v_{N}\right\|_{1}\right)\|h\|_{0} \\
& \leq C_{P} 2^{m-1} \kappa^{2}\left(1+2 c_{0}\right) N^{-m}+\frac{1}{2} c_{0}\left(\kappa+\left\|v_{N}\right\|_{1}\right)\|h\|_{0} .
\end{aligned}
$$

Now remembering that $\left\|v_{N}(\cdot, t)\right\|_{1} \leq \tilde{\beta}$ for $n \tilde{t} \leq t \leq(n+1) \tilde{t}$, and using Gronwall's inequality, the estimate

$$
\|h(\cdot, t)\|_{0} \leq\|h(\cdot, n \tilde{t})\|_{0} \mathrm{e}^{T c_{0}(\kappa+\tilde{\beta})}+C_{P} 2^{m-1} \kappa^{2}\left(1+2 c_{0}\right) N^{-m} T \mathrm{e}^{T c_{0}(\kappa+\tilde{\beta})}
$$

can be obtained. Next use the triangle inequality to conclude that

$$
\begin{aligned}
\left\|u(\cdot, t)-v_{N}(\cdot, t)\right\|_{0} \leq & \left\|u(\cdot, t)-P_{\frac{N}{2}} u(\cdot, t)\right\|_{0}+\left\|P_{\frac{N}{2}} u(\cdot, t)-v_{N}(\cdot, t)\right\|_{0} \\
\leq & C_{P} 2^{m} N^{-m}\|u(\cdot, t)\|_{m}+\|h(\cdot, t)\|_{0} \\
\leq & C_{P} 2^{m} N^{-m} \kappa+\|h(\cdot, n \tilde{t})\|_{0} \mathrm{e}^{T c_{0}(\kappa+\tilde{\beta})}+ \\
& +C_{P} 2^{m-1} \kappa^{2}\left(1+2 c_{0}\right) N^{-m} T \mathrm{e}^{T c_{0}(\kappa+\tilde{\beta})} \\
\leq & \left(C_{P} 2^{m} \kappa+C_{P} 2^{m-1} \kappa^{2}\left(1+2 c_{0}\right) T \mathrm{e}^{T c_{0}(\kappa+\tilde{\beta})}\right) N^{-m}+ \\
& +\left\|P_{N / 2} u(\cdot, n \tilde{t})-u(\cdot, n \tilde{t})\right\|_{0} \mathrm{e}^{T c_{0}(\kappa+\tilde{\beta})}+ \\
& +\left\|u(\cdot, n \tilde{t})-v_{N, 0}\right\|_{0} \mathrm{e}^{T c_{0}(\kappa+\tilde{\beta})} .
\end{aligned}
$$

Therefore, we have

$$
\begin{aligned}
& \sup _{t \in[n \tilde{t},(n+1) \tilde{t}]}\left\|u(\cdot, t)-v_{N}(\cdot, t)\right\|_{0} \\
& \leq\left(C_{P} 2^{m} \kappa+C_{P} 2^{m-1} \kappa^{2}\left(1+2 c_{0}\right) T \mathrm{e}^{T c_{0}(\kappa+\tilde{\beta})}\right) N^{-m}+ \\
& \quad+C_{P} 2^{m} N^{-m}\|u(\cdot, n \tilde{t})\|_{m} \mathrm{e}^{T c_{0}(\kappa+\tilde{\beta})}+\left\|u(\cdot, n \tilde{t})-v_{N, 0}\right\|_{0} \mathrm{e}^{T c_{0}(\kappa+\tilde{\beta})} .
\end{aligned}
$$

Thus choosing $\Lambda=C_{P} 2^{m} \kappa+C_{P} 2^{m-1} \kappa^{2}\left(1+2 c_{0}\right) T \mathrm{e}^{T c_{0}(\kappa+\tilde{\beta})}+C_{P} 2^{m} \kappa \mathrm{e}^{T c_{0}(\kappa+\tilde{\beta})}$ and $\lambda=\mathrm{e}^{T c_{0}(\kappa+\tilde{\beta})}$, the lemma is proved.

Corollary 3.4. With the same assumptions as in the previous lemma, there are constants $\tilde{\Lambda}_{m}$ and $\tilde{\lambda}_{m}$, such that the following estimate holds.

$$
\sup _{t \in[n \tilde{t},(n+1) \tilde{t}]}\left\|u(\cdot, t)-v_{N}(\cdot, t)\right\|_{1} \leq \tilde{\Lambda} N^{1-m}+\tilde{\lambda} N\left\|u(\cdot, n \tilde{t})-v_{N, 0}\right\|_{0} .
$$


Proof. The proof follows from the triangle inequality and the inverse inequality (1.7).

$$
\begin{aligned}
\left\|u(\cdot, t)-v_{N}(\cdot, t)\right\|_{1} \leq & \left\|u(\cdot, t)-P_{N / 2} u(\cdot, t)\right\|_{1}+\left\|P_{N / 2} u(\cdot, t)-v_{N}(\cdot, t)\right\|_{1} \\
\leq & C_{P} 2^{m-1} N^{1-m}\|u(\cdot, t)\|_{m}+N\left\|P_{N / 2} u(\cdot, t)-v_{N}(\cdot, t)\right\|_{0} \\
\leq & C_{P} 2^{m-1} N^{1-m} \kappa+N\|h(\cdot, t)\|_{0} \\
\leq & C_{P} 2^{m-1} N^{1-m} \kappa+N\|h(\cdot, n \tilde{t})\|_{0} \mathrm{e}^{T c_{0}(\kappa+\tilde{\beta})}+ \\
& +C_{P} 2^{m-1} \kappa^{2}\left(1+2 c_{0}\right) N^{1-m} T \mathrm{e}^{T c_{0}(\kappa+\tilde{\beta})} \\
\leq & C_{P} 2^{m-1} N^{1-m} \kappa+N\left\|v_{N, 0}-u(\cdot, n \tilde{t})\right\|_{0} \mathrm{e}^{T c_{0}(\kappa+\tilde{\beta})}+ \\
& +N\left\|u(\cdot, n \tilde{t})-P_{N / 2} u(\cdot, n \tilde{t})\right\|_{0} \mathrm{e}^{T c_{0}(\kappa+\tilde{\beta})}+ \\
& +C_{P} 2^{m-1} \kappa^{2}\left(1+2 c_{0}\right) N^{1-m} T \mathrm{e}^{T c_{0}(\kappa+\tilde{\beta})} .
\end{aligned}
$$

Choosing the constants $\tilde{\Lambda}$ and $\tilde{\lambda}$ appropriately, the result follows.

In order to keep the exposition as tidy as possible, we take $\Lambda$ to be the maximum of $\Lambda$ and $\tilde{\Lambda}$, and $\lambda$ to be the maximum of $\lambda$ and $\tilde{\lambda}$. The results obtained so far are now put together to prove the following theorem.

TheOrem 3.5. Suppose $u \in C\left([0, T], H_{\#}^{m}\right)$ is a solution of (1.8) with initial data $u_{0} \in H_{\#}^{m}(0,2 \pi)$, where $m \geq 2$. Then for $N$ large enough, there exist solutions $v_{N} \in C\left([0, T], S_{N}\right)$ of (3.3) with initial data $I_{N} u_{0}$. Moreover, there exists a constant $C^{*}$ depending on $\left\|u_{0}\right\|_{m}$ and $T$, such that

$$
\sup _{t \in[0, T]}\left\|u(\cdot, t)-v_{N}(\cdot, t)\right\|_{1} \leq C^{*} N^{1-m} .
$$

Proof. The strategy of the proof is to apply the local error analysis $q$ times, thereby piecing together a solution on the interval $[0, T]$. Given $\left\|u_{0}\right\|_{m}$ and the final time $T$, Theorem 2.1 provides the constant $\kappa$, so that $\|u(\cdot, t)\|_{m} \leq \kappa$, for $0 \leq t \leq T$. Choose $R=2 \kappa$; then Corollary 3.2 yields a time $\tilde{t}$, such that the approximate equation can be solved on the interval $[n \tilde{t},(n+1) \tilde{t}]$. Let $q$ be an integer larger than $T / \tilde{t}$. The convergence will hold for $N$ so large that

$$
N^{1-m} \Lambda \sum_{l=0}^{q-1} \lambda^{l}+N^{1-m} \lambda^{q} C_{I}\left\|u_{0}\right\|_{m}<\kappa .
$$

To obtain an approximation on the interval $[0, \tilde{t}]$, we solve (3.6) with $n=0$ and initial data $v_{N, 0}=I_{N} u_{0}$. It follows from the preceding results that we have the error estimates

$$
\sup _{t \in[0, \tilde{t}]}\left\|u(\cdot, t)-v_{N}(\cdot, t)\right\|_{0} \leq \Lambda N^{-m}+\lambda\left\|u_{0}-I_{N} u_{0}\right\|_{0}
$$

and

$$
\sup _{t \in[0, \tilde{t}]}\left\|u(\cdot, t)-v_{N}(\cdot, t)\right\|_{1} \leq \Lambda N^{1-m}+\lambda N\left\|u_{0}-I_{N} u_{0}\right\|_{0} .
$$


Moreover, we obtain the following inequality

$$
\begin{aligned}
\left\|v_{N}(\cdot, t)\right\|_{1} & \leq\left\|v_{N}(\cdot, t)-u(\cdot, t)\right\|_{1}+\|u(\cdot, t)\|_{1} \\
& \leq \Lambda N^{1-m}+\lambda N\left\|u_{0}-I_{N} u_{0}\right\|_{0}+\kappa \\
& \leq \Lambda N^{1-m}+\lambda N^{1-m} C_{I}\left\|u_{0}\right\|_{m}+\kappa,
\end{aligned}
$$

for $t \in[0, \tilde{t}]$.

By (3.10), it follows that $\left\|v_{N}(\cdot, \tilde{t})\right\|_{1} \leq 2 \kappa=R$, so that the foregoing reasoning can be reapplied, and the solution be continued to the interval $[\tilde{t}, 2 \tilde{t}]$. In other words, Equation (3.6) can be posed with $n=1$ and initial data $v_{0, N}=v_{N}(\cdot, \tilde{t})$, and compared with the solution of Equation (1.8) on the interval $[\tilde{t}, 2 \tilde{t}]$. Since $\left\|v_{N}(\cdot, \tilde{t})\right\|_{1} \leq R$, the time of existence $\tilde{t}$ and the bound $\tilde{\beta}$ are the same as during the first step, and the constants $\Lambda$ and $\lambda$ are the same as before. Accordingly, the following error estimates hold.

$$
\begin{aligned}
\sup _{t \in[\tilde{t}, 2 \tilde{t}]}\left\|u(\cdot, t)-v_{N}(\cdot, t)\right\|_{0} & \leq \Lambda N^{-m}+\lambda \Lambda\left\|u(\cdot, \tilde{t})-v_{N}(\cdot, \tilde{t})\right\|_{0} \\
& \leq \Lambda N^{-m}+\lambda \Lambda N^{-m}+\lambda_{m}^{2} N^{-m} C_{I}\left\|u_{0}\right\|_{m}, \\
\sup _{t \in[\tilde{t}, \tilde{t}]}\left\|u(\cdot, t)-v_{N}(\cdot, t)\right\|_{1} & \leq \Lambda N^{1-m}+\lambda \Lambda N \| u\left(\cdot, \tilde{t}-v_{N}(\cdot, \tilde{t}) \|_{0}\right. \\
& \leq \Lambda N^{1-m}+\lambda \Lambda N^{1-m}+\lambda_{m}^{2} N^{1-m} C_{I}\left\|u_{0}\right\|_{m} .
\end{aligned}
$$

Moreover we have the estimate

$$
\begin{aligned}
\sup _{t \in[\tilde{t}, 2 \tilde{t}]}\left\|v_{N}(\cdot, t)\right\|_{1} & \leq\left\|v_{N}(\cdot, 2 t)-u(\cdot, 2 t)\right\|_{1}+\|u(\cdot, 2 t)\|_{1} \\
& \leq \Lambda N^{1-m}+\lambda \Lambda N^{1-m}+\lambda_{m}^{2} N^{1-m} C_{I}\left\|u_{0}\right\|_{m}+\kappa \\
& \leq R .
\end{aligned}
$$

To continue the solution to the interval $[0, T]$, remember that $\tilde{t}$ only depends on $R$, which fact assures that the local analysis can be applied $q$ times on an interval of length $\tilde{t}$ to yield a solution $v_{N}$ on the interval $[0, T]$. The requirement (3.10) assures that the solution can be continued further each time. At the $n$-th stage, we have the estimates

$$
\begin{aligned}
& \sup _{t \in[n \tilde{t},(n+1) \tilde{t}]}\left\|u(\cdot, t)-v_{N}(\cdot, t)\right\|_{0} \leq N^{-m} \Lambda \sum_{l=0}^{n-1} \lambda^{l}+N^{-m} \lambda^{n} C_{I}\left\|u_{0}\right\|_{m}, \\
& \sup _{t \in[n \tilde{t},(n+1) \tilde{t}]}\left\|u(\cdot, t)-v_{N}(\cdot, t)\right\|_{1} \leq N^{1-m} \Lambda \sum_{l=0}^{n-1} \lambda^{l}+N^{1-m} \lambda^{n} C_{I}\left\|u_{0}\right\|_{m},
\end{aligned}
$$

and

$$
\begin{aligned}
\sup _{t \in[n \tilde{t},(n+1) \tilde{t}]}\left\|v_{N}(\cdot, t)\right\|_{1} & \leq N^{1-m} \Lambda \sum_{l=0}^{n-1} \lambda^{l}+N^{1-m} \lambda^{n} C_{I}\left\|u_{0}\right\|_{m}+\kappa \\
& \leq R .
\end{aligned}
$$


Since the local error estimate holds on each subinterval, it also holds on the interval $[0, T]$ with the constant $C^{*}=\Lambda \sum_{l=0}^{q-1} \lambda^{l}+\lambda^{q} C_{I}\left\|u_{0}\right\|_{m}$.

\section{Numerical implementation.}

In this section, an implementation of the discretization procedure described above is used to confirm the convergence results experimentally. As an application of the numerical method explained in this paper, the interaction of two solitary waves for the regularized Benjamin-Ono equation on the real line is investigated.

To reach a fully discrete approximation, the Fourier-collocation projection is coupled with a 4-stage Runge-Kutta time-stepping algorithm. The 4-stage Runge-Kutta method is a natural choice for this problem, because the resulting system of ordinary differential equations is not stiff. Moreover, since the spectral method is rapidly convergent, it is natural to use a time discretization of fairly high order.

For the periodic regularized Benjamin-Ono equation, there are exact solutions in the form of a traveling waves. To find such a solution, assume that a solution of (1.3) has the form

$$
u(x, t)=\psi(x-c t),
$$

where the function $\psi$ is $2 \pi$-periodic. Substituting this expression into (1.3) and integrating, there appears the equation

$$
-c \psi+\psi+\frac{1}{2} \psi^{2}-c H \psi^{\prime}
$$

A $2 \pi$-periodic solution of this integro-differential equation is given by the formula

$$
u(x, t)=\frac{c \sinh (p)}{\cosh ^{2}\left(\frac{p}{2}\right)-\cos ^{2}\left(\frac{x-c t}{2}\right)},
$$

with the speed

$$
c=\frac{\tanh (p)}{\tanh (p)-1} .
$$

A plot of a typical periodic traveling wave is provided in Figure 4.1. These solutions can be used to test the numerical algorithm, because their time evolution is simply given by translation. If $u_{N}\left(\cdot, t_{i}\right)$ is the result of a numerical computation with initial data $\psi(x)$, it can be compared with the translated function $\psi\left(x-c t_{i}\right)$. In this way, the error produced by the discretization can be calculated. In particular, the convergence rate of the scheme can be found. In Table 4.1, a traveling wave with $p=0.5$ was used, so that the wave speed $c$ was approximately -0.85914 . Table 4.1 shows the error due to the time discretization when $N=8192$. It appears that the formal 4-th order convergence is approximately achieved. Table 4.1 also shows the error due to the space discretization with the time step $\delta=0.0002$. It is apparent that the error goes down exponentially until it approaches machine precision, confirming the spectral convergence of the 


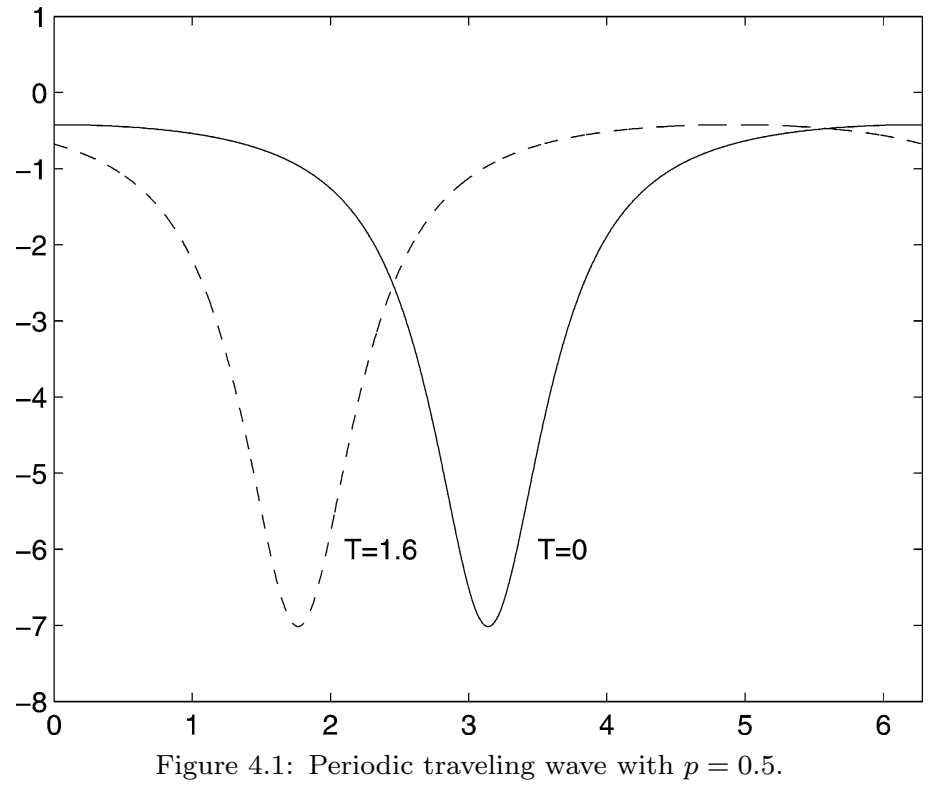

Table 4.1: Discretization error for the traveling wave in (4.2) with $p=0.5$ over the time domain $[0,1.6]$. In the left table, the number of grid points is $N=8192$. In the right table, the time step is $\delta=0.0002$.

\begin{tabular}{|l|c|r|}
\hline$\delta$ & $L^{2}$-Error & \multicolumn{1}{|c|}{ Ratio } \\
\hline 0.4000 & $3.1362 \mathrm{e}-02$ & \\
0.2000 & $3.7388 \mathrm{e}-03$ & 8.39 \\
0.1000 & $2.0942 \mathrm{e}-04$ & 17.85 \\
0.0500 & $1.1072 \mathrm{e}-05$ & 18.92 \\
0.0250 & $6.1732 \mathrm{e}-07$ & 17.94 \\
0.0125 & $3.6156 \mathrm{e}-08$ & 17.08 \\
0.0063 & $2.1833 \mathrm{e}-09$ & 16.56 \\
0.0031 & $1.3407 \mathrm{e}-10$ & 16.29 \\
0.0016 & $8.3043 \mathrm{e}-12$ & 16.14 \\
0.0008 & $5.1674 \mathrm{e}-13$ & 16.07 \\
\hline
\end{tabular}

\begin{tabular}{|l|r|r|}
\hline$N$ & $L^{2}$-Error & Ratio \\
\hline & & \\
& & \\
8 & $8.0646 \mathrm{e}-01$ & 0 \\
16 & $3.8072 \mathrm{e}-02$ & 21.18 \\
32 & $6.3915 \mathrm{e}-04$ & 59.57 \\
64 & $2.1651 \mathrm{e}-07$ & $2,952.00$ \\
128 & $2.4899 \mathrm{e}-14$ & $8,696,000.00$ \\
256 & $1.7745 \mathrm{e}-15$ & 14.03 \\
& & \\
\hline
\end{tabular}

collocation scheme. Similar results hold for other values of the parameter $p$, and it can be said in conclusion that the Runge-Kutta method provides an excellent companion to the spectral method.

To conclude our study, we simulate the interaction of two solitary waves. Solitary waves are solutions of Equation (4.1) posed on the real line which have decay at infinity. These solutions are given by the expression

$$
u(x, t)=\frac{4 d}{1+\left(\frac{d}{d+1}\right)^{2}(x-(d+1) t)^{2}},
$$


Table 4.2: Discretization error for the solitary wave in (4.3) with $d=0.75$. In the left table, the error at $T=1000$ is shown. In the right table, the error at $T=2000$ is shown. The number of gridpoints is $N=16384$.

\begin{tabular}{|l|c|r|l|c|}
\hline & \multicolumn{2}{|c|}{$T=1000$} & \multicolumn{2}{c|}{$T=2000$} \\
\hline$\delta$ & $L^{\infty}$-Error & Ratio & $L^{\infty}$-Error & Ratio \\
\hline 0.1000 & $2.9786 \mathrm{e}-02$ & & $1.1682 \mathrm{e}-01$ & \\
0.0500 & $9.7420 \mathrm{e}-04$ & 30.6 & $3.7770 \mathrm{e}-03$ & 30.9 \\
0.0250 & $3.3149 \mathrm{e}-05$ & 29.4 & $1.2832 \mathrm{e}-04$ & 29.4 \\
0.0125 & $1.9109 \mathrm{e}-06$ & 17.3 & $1.0481 \mathrm{e}-05$ & 12.2 \\
0.0063 & $9.9190 \mathrm{e}-07$ & 1.9 & $8.9896 \mathrm{e}-06$ & 1.2 \\
\hline
\end{tabular}
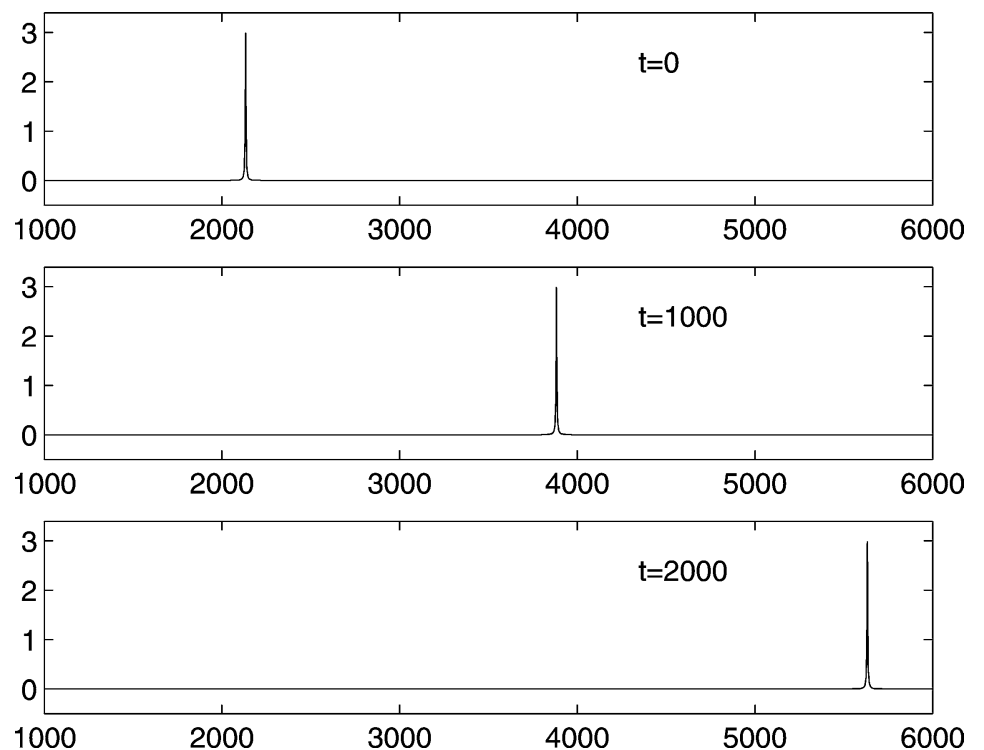

Figure 4.2: Solitary-wave solution of the regularized Benjamin-Ono equation with $d=0.75$.

where the speed of translation of the solitary wave is $d+1$. Since the Fouriercollocation scheme approximates the problem with periodic boundary conditions, the quadratic decay of this function can be a problem if not a sufficiently large spatial domain is used. In the calculations shown here the size of the domain $[0, L]$ was at least $L=6400$.

The evolution of a single solitary wave of height 3 is shown in Figure 4.2. As it appears, the scheme produces a small error even if integrating over a rather large time interval. It can be seen from Table 4.2 that for a integration up to $T=2000$, with $N=16384$ gridpoints, a time step of $\delta=0.0125$ is appropriate.

To initiate the interaction, a solitary wave of height 3 and a solitary wave of height 6 were superimposed. The larger solitary wave was placed to the left of the smaller wave. They were arranged so that they were initially separated by 

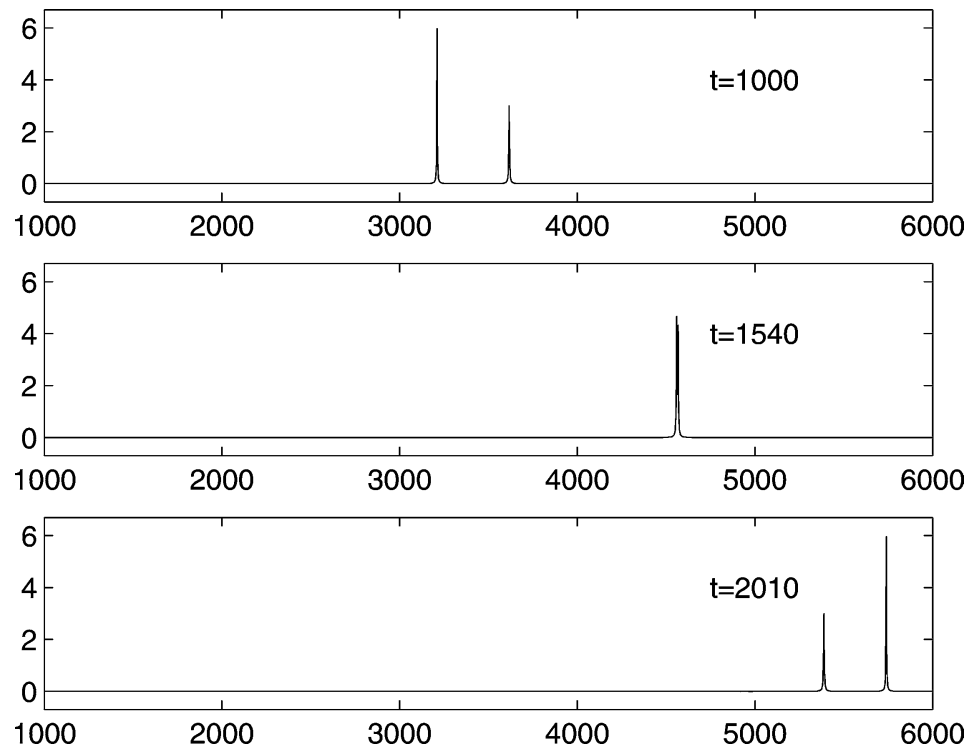

Figure 4.3: Interaction of two solitary waves for the regularized Benjamin-Ono equation.
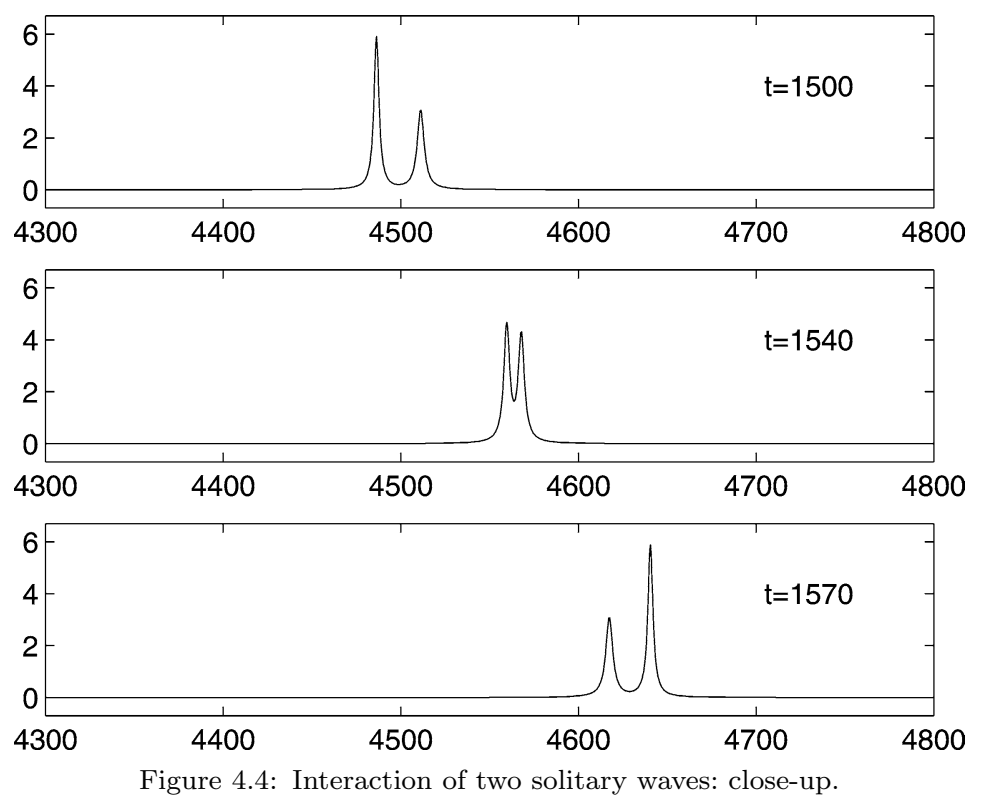

about 1600 , which was enough to bring the overlap down to about $10^{-5}$. The domain was chosen large enough, so that the tails of the solitary waves were of magnitude $10^{-5}$ at the boundary, and that the evolution could be followed without one of the waves wrapping around and reentering at the other end. The two solitary waves coalesced at about $t=1450$, and the larger wave then 


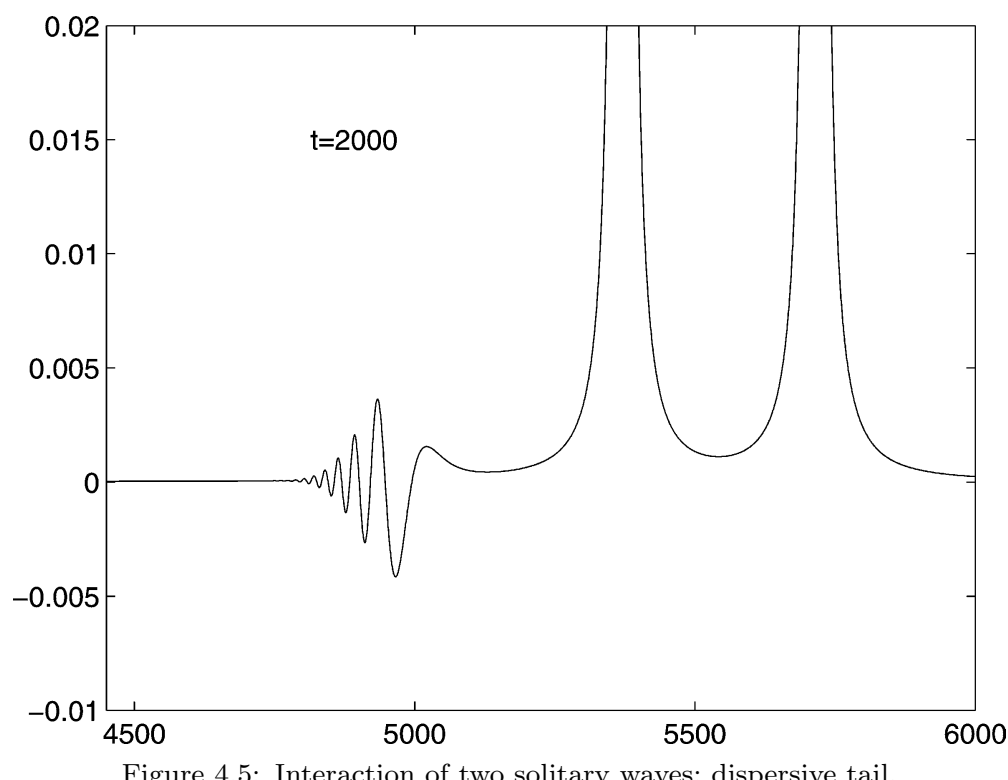

passed the smaller one. It can be seen in Figure 4.5 that after the waves pulled apart, a small trailing wave train appeared. Since the tail is on the order of $10^{-3}$, and the $L^{\infty}$-Error for the evolution of a single solitary wave was on the order of $10^{-5}$ at $T=2000$, the dispersive tail does not appear to be a numerical artifact. To be sure, the experiments were conducted with varying domain sizes, and varying initial separation of the solitary waves. This did not have an effect on the interaction. In particular, the height of the dispersive tail that appeared after the interaction did not change significantly. Varying spatial and temporal grid sizes did not affect the results either.

\section{Acknowledgement.}

The author wishes to thank Beatrice Pelloni for pointing out the problem treated in this article.

\section{REFERENCES}

1. J. P. Albert and J. L. Bona, Comparisons between model equations for long waves, J. Nonlinear Sci., 1 (1991), pp. 345-374.

2. T. B. Benjamin, Internal Waves of permanent form in fluids of great depth, J. Fluid Mechanics, 29 (1967), pp. 559-592.

3. C. Canuto, M. Y. Hussaini, A. Quarteroni, and T. A. Zang, Spectral Methods in Fluid Dynamics, Springer, 1988.

4. H. Kalisch and J. L. Bona, Models for internal waves in deep water, Disc. Cont. Dyn. Sys., 6 (2000), pp. 1-19.

5. H.-O. Kreiss and J. Oliger, Stability of the Fourier method, SIAM J. Numer. Anal., 16 (1979), pp. 421-433.

6. Y. Maday and A. Quarteroni, Error analysis for spectral approximation of the Kortewegde Vries equation, RAIRO Modl. Math. Anal. Numr., 22 (1988), pp. 499-529. 
7. H. Ono, Algebraic solitary waves in stratified fluids, J. Phys. Soc. Japan, 39 (1975), pp. 1082-1091.

8. J. E. Pasciak, Spectral and pseudospectral methods for advection equations, Math. Comput., 35 (1980), pp. 1081-1092.

9. J. E. Pasciak, Spectral methods for a nonlinear initial value problem involving pseudodifferential operators, SIAM J. Numer. Anal., 19 (1982), pp. 142-153.

10. B. Pelloni and V. A. Dougalis, Numerical solution of some nonlocal, nonlinear dispersive wave equations, J. Nonlinear Sci., 10 (2000), pp. 1-22.

11. B. Pelloni and V. A. Dougalis, Error estimates for a fully discrete spectral scheme for a class of nonlinear, nonlocal dispersive wave equations, Appl. Numer. Math., 37 (2001), pp. $95-107$.

12. V. Thomeé and A. S. Vasudeva Murthy, A numerical method for the Benjamin-Ono equation, BIT, 38 (1998), pp. 597-611. 